Écrire

l'histoire

\section{Écrire l'histoire}

Histoire, Littérature, Esthétique

12 | 2013

Présent (2)

\title{
Écouter l'histoire ou la clé des luttes passées
}

Sur le générique du Fond de l'air est rouge, de Chris Marker (1977)

\section{Vincent Casanova}

\section{(2) OpenEdition}

Journals

Édition électronique

URL : http://journals.openedition.org/elh/341

DOI : $10.4000 /$ elh.341

ISSN : 2492-7457

Éditeur

CNRS Éditions

\section{Édition imprimée}

Date de publication : 15 novembre 2013

Pagination : 75-81

ISBN : 978-2-35698-065-6

ISSN : 1967-7499

Référence électronique

Vincent Casanova, «Écouter l'histoire ou la clé des luttes passées », Écrire l'histoire [En ligne], 12 | 2013, mis en ligne le 15 novembre 2016, consulté le 23 septembre 2020. URL : http://

journals.openedition.org/elh/341 ; DOI : https://doi.org/10.4000/elh.341 


\section{Écouter l'histoire ou la clé des luttes passées}

\section{Sur le générique du Fond de l'air est rouge, de Chris Marker (1977)}

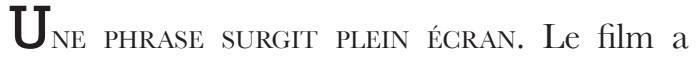
commencé. Le titre s'affiche, à nu, devant nos yeux: «LE FOND DE L'AIR EST ROUGE ». C'est en lettres capitales découpées, en rouge sur un fond noir ${ }^{1}$. Les caractères ont une unité formelle. Les lettres semblent naître les unes des autres. La typographie est simple. Elle alterne et combine, par le hasard des mots, lignes verticales, horizontales et courbes, sans empattement, dessinant comme un slogan qu'on aurait soigneusement imprimé. L’ensemble a en réalité émergé subrepticement, depuis et autour d'une masse circulaire pleine et rouge se rétractant fugitivement, revenant pour faire office de première voyelle du mot «ROUGE». En revanche, l'autre « $\mathrm{O}$ » du titre a été laissé vide et ne forme qu'un cercle fin: ces jeux visuels, légers et fugaces, signalent la dimension singulière du « $\mathrm{O}$ ». Arrondi spectaculaire dans un environnement dominé par des formes orthogonales, ce signe pris dans un signifiant - rouge de colère et rouge sang, rouge passion et rouge politique - a une grande puissance d'évocation et d'association. On songe notamment au soleil dont le Japon ${ }^{2}$ a fait

Vincent Casanova, université Paris-I Panthéon-Sorbonne.

1. L'analyse se fonde sur la version du film qui a été éditée en DVD en 2008 par Arte, Iskra et l'INA. Cette version comprend des différences avec la version initiale. Cf. Chris Marker, Le Fond de l'air est rouge. Scènes de la Troisième Guerre mondiale, 1967-1977. Textes et description d'un film de Chris Marker, François Maspero (Voix), 1978.

2. Un des terrains d'exploration favoris de Marker. 


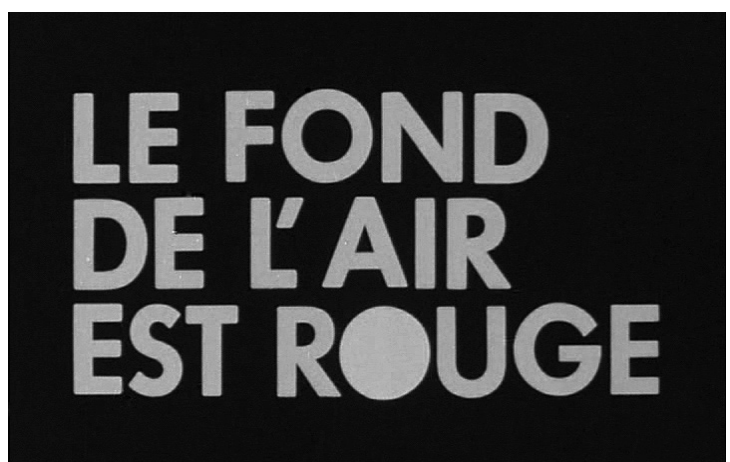

Le titre du film ou l'image-générique.

son drapeau. Mais cette «image-générique ${ }^{3}$ » évoque aussi un crépuscule: le cercle rouge flotte dans un fond noir, le titre éveille un imaginaire qu'on associe au (grand!) soir. Et pour cause: le film documente les mouvements révolutionnaires qui traversent le monde.

Marker est coutumier du fait: poser une image d'une part pour énoncer d'autre part. Sauf qu'il procède là à une condensation remarquable. La déclaration inaugurale, au-delà de sa transparence grammaticale, est une figure: les mots eux-mêmes font image. Plus significativement peut-être, les « $\mathrm{O}$ » se suivent et ne se ressemblent pas. Or ce « cercle déporté à l'infini ${ }^{4}$ »n'est pas une forme anodine pour Marker; ce n'est rien d'autre que la métaphore

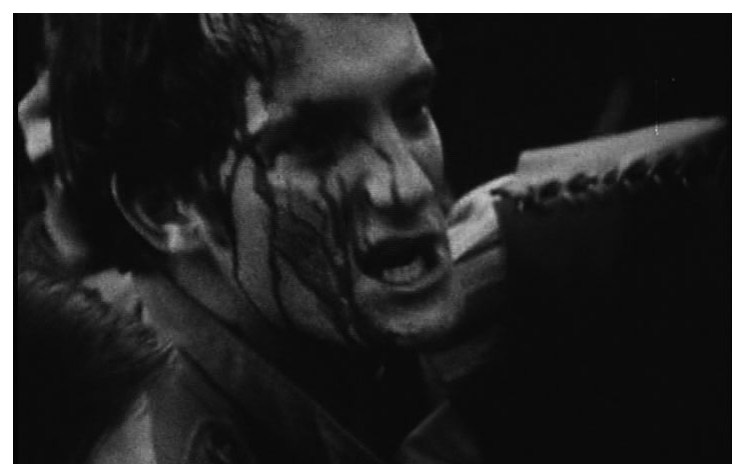

Le visage ensanglanté d'un manifestant anonyme dans sa lutte contre les Pouvoirs.

du temps, comme le déclare la voix off de Sans Soleil:

Dans la spirale du générique [de Vertigo de Hitchcock], il voyait le Temps qui couvre un champ de plus en plus large. ${ }^{5}$

Autrement dit, rien ne change, et tout est différent: le soleil a beau se coucher, l'histoire continue sa révolution. Tout semble dit. Prolongeons pourtant. À la suite de cette genèse, entre écrit et image, le générique déroule la matrice telle une spirale. C'est à celui-ci que les lignes qui suivent sont consacrées. Repartons du début.

Le carton-titre s'affiche. Puis une voix off survient: celle de Simone Signoret, qui parle à la première personne. L'actrice semble répondre à une question. Cette voix se souvient du Cuirassé

3. Philippe Dubois (dir.), «La Fetée ou le cinématogramme de la conscience », Théorème, n 6, « Recherches sur Chris Marker », 2002, p. 8-45 (p. 13-17).

4. La définition de la spirale que donne Roland Barthes dans «Réquichot et son corps » [1973], in Euvres complètes, t. II, Éd. du Seuil, 1997, p. 94.

5. Sans Soleil (1982), $67^{\mathrm{e}}$ minute. 


\section{Musique du générique LUCIANO BERIO}

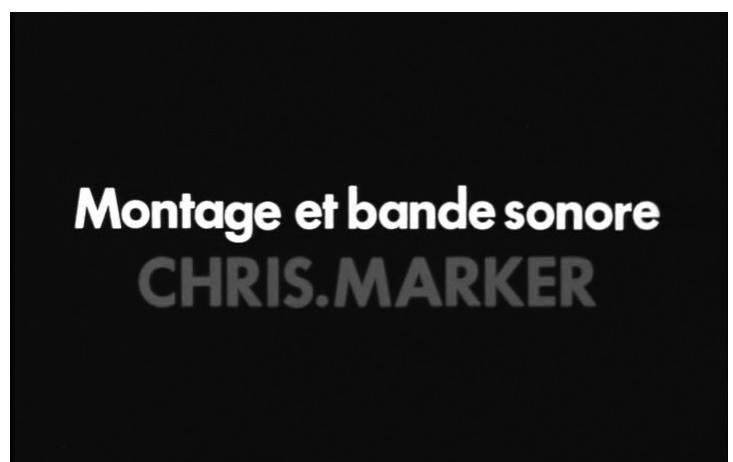

Le second carton: un cinéaste en habit de compositeur.

voix se tait pour laisser place à une musique et à un montage d'images qui fait alterner des scènes de luttes sociales des années 1960 et des plans saisissants du film d'Eisenstein. C'est là le dispositif d'ouverture du film. Le générique dure quatre minutes. La musique va crescendo jusqu'à son arrêt soudain avec l'entretien de la guide de l'Intourist ${ }^{7}$ sur l'escalier d'Odessa où se rompt la mécanique musicale.

On voudrait rendre justice à celle-ci. D’une part parce que Marker lui-même a reconnu un modèle musical dans sa pratique ${ }^{8}$. D'autre part parce que Le Fond de l'air est rouge marque un tournant dans le travail sonore de Marker: celui-ci, pour la première fois, réalise des sons électroniques grâce à

6. Le mot est écrit en cyrillique.

7. L'unique agence à laquelle les touristes avaient affaire pour voyager en URSS.

8. Dans le dossier de presse de Sans Soleil, titre qu'il emprunte à un cycle de mélodies du compositeur russe Moussorgski (18391881), Marker déclare : «Un cinéaste [...] préfère livrer les pièces du dossier à la façon d’une composition musicale, avec thèmes récurrents, contrepoints et fugues en miroir. » Cité par Arnaud Lambert, Also known as Chris Marker, Cherbourg, Le Point du jour, 2008, p. 214.

Vincent Casanova, «Écouter l'histoire ou la clé des luttes passées. Sur le générique du Fond de l'air est rouge, de Chris Marker (1977) » 
des synthétiseurs pour la bande sonore d'un de ses films. Aussi est-ce la notion double de «montage et bande sonore » qui est associée au nom de Marker dans ce générique d'ouverture. Significativement, toutefois, l'a précédé le nom du compositeur italien Luciano Berio (1925-2003), crédité de la musique du générique.

La partition de Berio a été primitivement conçue comme « une sorte de blague pour des amis à La Scala ${ }^{9} »$. Contemporaine du film, cette brève œuvre symphonique, créée à l'opéra de Milan en 1975, est une transcription - ou plutôt le montage sous la forme d'une réorchestration - d'une œuvre écrite par Luigi Boccherini (1743-1805), compositeur italien installé en Espagne à la fin du XVIII ${ }^{\mathrm{e}}$ siècle. À l'origine, il y a le mouvement d'un quintette à cordes de Boccherini intitulé La Musica notturna delle strade di Madrid. Le succès rencontré par l'œuvre conduisit Boccherini à en faire des versions différentes, avec guitare, avec harpe, avec piano. Puis, il la reprit pour en faire le troisième mouvement du sixième des quintettes avec piano (opus 57, posthume) qu’il dédia en 1799 à la nation française « en témoignage de la vive reconnaissance et gratitude qu'[il] éprouv[ait] à l'égard de cette grande nation ${ }^{10}$ ".

D'inspiration populaire, le thème, de facture classique, est simple et court, facilement mémorisable, fondé sur une cellule mélodico-rythmique à partir de laquelle l'ensemble du discours musical est élaboré ${ }^{11}$. La mesure est à deux temps, un rythme pointé ainsi qu'un saut intervallique donnent de l'élan, le tempo est modéré, les hauteurs sont le plus souvent conjointes, l'assise tonale est affirmée en ut majeur. Le mouvement d'ensemble est celui d'une marche: dans le programme conçu par Boccherini pour son quintette, il s'agit d'une sérénade nocturne jouée par des musiciens joyeux marchant dans la rue et s'éloignant dès qu'ils entendent les appels du gardien de nuit. C'est ce déplacement dans la ville qui donne sa forme à l'œuvre: il s'agit d'un thème et variations, celles-ci incarnant le cheminement des musiciens dans l'espace depuis le point d'écoute (fixe) de l'auditeur; aussi la musique va-t-elle crescendo-decrescendo. La mélodie est répétée obstinément, mais à chaque fois sous une forme différente car l'instrumentation, les harmonies, les nuances, l'ornementation, changent. L'ensemble constitue par son intensité une arche sonore: la fin se termine dans la même nuance piano du début.

Le travail de Berio a consisté à superposer quatre des versions existantes composées par Boccherini et à arranger le tout, en ajoutant simplement une courte introduction de huit mesures (inaudible dans

9. Luciano Berio cité par Ivanka Stoïanova, «Luciano Berio. Chemins en musique », La Revue musicale, n ${ }^{\text {os }} 375-377$, Éd. Richard Masse, 1985, p. 457.

10. Cf. Yves Gérard, plaquette du CD Luigi Boccherini. Trois quintetti dédiés à la Nation française, opus 57, interprétés par Patrick Cohen et le Quatuor Mosaïques, Astrée E 8721.

11. Cf. ci-après le schéma d'analyse du thème musical. Mes remerciements à Louise Bernard de Raymond pour la mise en forme. 
le générique du film). On peut donc considérer que Berio n'est pas à strictement parler l'auteur de la musique, dans la mesure où il a arrangé un matériau musical déjà existant. Il l'a dit lui-même:

Il s'agit d'une transcription de différentes transcriptions. Évidemment, j’ai dû parfois adapter les choses. ${ }^{12}$

Or on ne saurait mieux définir le travail de Marker pour Le Fond de l'air est rouge puisque les images qui composent le film n'ont pas été tournées par Marker lui-même, comme il le rappelle dans le générique de fin, mais par d'«innombrables cameramen, preneurs de son, témoins et militants ».

Gette identité dans la démarche de montage et de collage se rejoue dans l'exceptionnelle coïncidence de l'image et du son: des hommes défilent (au son de grosses caisses parfois comme le laisse voir une image) dans les rues du monde entier et l'on entend le roulement des percussions (ajoutées par Berio mais dont l'effet était souhaité par Boccherini). C'est dans cette émulation réciproque que réside en partie l'émotion produite par le générique sur le spectateur. Ainsi tout le début du générique repose-t-il sur la conjonction d'images fortes soutenues par une musique à l'efficacité indéniable. L'adéquation entre le son et l'image tient aussi à l'équivalence de leur intensité. En effet, plus la musique va crescendo, plus les manifestations se transforment en affrontements violents. Plus que les images, c'est bien la musique qui prend en charge la progression dramatique. Marker suit la forme induite par l'œuvre musicale.
Aussi est-ce la musique dans sa forme même qui prend en charge la direction du film.

Le thème musical se décompose en deux temps: \{A: a-b $\}$ constitue l'antécédent; $\left\{\mathrm{A}^{\prime}: \mathrm{a}^{\prime}-\mathrm{b}\right\}$, le conséquent.

\section{ANTÉCÉDENT: A}



Or Marker conçoit aussi son générique en deux parties: il l'organise symétriquement autour des six premières variations de l'œuvre de Berio (trois pour les artistes mentionnés, trois sans inserts). Il redéploie cette bipartition également pour l'ensemble du film: celui-ci se compose de deux grandes parties, chacune se décomposant en deux sous-parties. Dès lors la première partie du film, « Les Mains fragiles », constitue l'antécédent et la seconde partie, « Les Mains coupées », son conséquent. Le générique apparaît ainsi comme la miniature du plan d'ensemble du film. En conservant seulement la première moitié du thème et variations pour son générique, Marker opère un geste qu'il reste à élucider.

Tout le film de Marker met en scène une césure historique; c'està cette aune qu'il convient d'analyser

12. Ivanka Stoïanova, op. cit., p. 457.

Vincent Casanova, «Écouter l'histoire ou la clé des luttes passées. Sur le générique du Fond de l'air est rouge, de Chris Marker (1977) »

Écrire l'histoire, $\mathrm{n}^{\circ} 12$ - automne 2013, 75-81 
son utilisation de la musique de Berio. Le cinéastemonteur « coupe »leson eninterrompantla musique à son point culminant, oblitérant la deuxième partie de l'œuvre dans laquelle Boccherini-Berio ménageaient un decrescendo équivalent au crescendo. En interrompant l'œuvre de Boccherini-Berio au climax, au point d'incandescence maximale, Marker, en cette fin de générique, fait entendre la musique par les images: tout le film procède par synesthésie.

Marker le présente ainsi:

Interroger en quelque sorte, autour d'un thème précis (l'évolution de la problématique politique dans le monde autour des années 1967-1970), notre refoulé en images. ${ }^{13}$

Or le refoulé, c'est tout à la fois ce qui reflue et ce qui est supposé disparaître, comme les chutes de bobines dont le film est constitué et qui ont capté tout au long de ces années la « longue suite de défaites ${ }^{14}$ » que connaissent les mouvements d'émancipation dans le monde. Souvenons-nous de l'«imagegénérique " qui évoquait au commencement le crépuscule, le couchant des luttes. Le générique réunit mots, couleurs, paroles, images et sons en un seul et même mouvement, chacun se faisant très directement écho, parvenant à mettre en résonance une multiplicité de formes d'expression. Marker a bien composé une œuvre symphonique: il fait
« sonner ensemble ». Le montage doit restituer « à l'histoire sa polyphonie ${ }^{15}{ }$. Est-ce une coïncidence si la musique de Berio manifeste la quintessence d'un travail polyphonique? Assembler, superposer des strates, des couches sonores, c'est là la définition minimale mais essentielle de la polyphonie. C'est pourquoi elle paraît aussi à l'image même du film: cette entreprise de mise en cohérence des différentes luttes, unissant la pluralité et l'hétérogénéité des gauches, est à l'œuvre au cœur de la musique.

En outre, cette œuvre permet à Marker de substituer aux hymnes «officiels» de la révolution une mélodie vierge de toute récupération politique. Elle opère par la magie compositionnelle de Berio une jonction entre passé et présent. Marker se sert de la musique pour faire entendre l'histoire telle qu'il la comprend:

Au cours des dix dernières années, un certain nombre d'hommes et de forces (quelquefois plus instinctives qu'organisées) ont tenté de jouer pour leur compte - fût-ce en renversant les pièces. Tous ont échoué sur les terrains qu'ils avaient choisis. C'est quand même leur passage qui a le plus profondément transformé les données politiques de notre temps. ${ }^{16}$

Autrement dit, une fois encore, rien n'a changé, mais tout est différent. C'est un peu aussi le sens du travail de transcriptions, d'orchestration de Berio. Sans compter que, par la forme du thème

13. Chris Marker, op. cit., p. 5.

14. Ibid., p. 6.

15. Ibid.

16. Ibid., $4^{\mathrm{e}}$ de couverture (elle est signée Chris. Marker). 
et variations en crescendo et decrescendo, la musique s'enroule sur elle-même telle une spirale.

Pour Marker, le présent correspond au centre de la spirale, à son œil, et le passé en extension est figuré par les spires. Ainsi le temps est-il un composé de passé et de présent. Le futur, lui, est comme aspiré par l'œil de la spirale. C'est là la conception de l'histoire de Marker: l'histoire - le passé - se fait toujours au présent et configure le futur.

C'est un jeu bizarre, dont les règles changent au fur et à mesure de la partie. ${ }^{17}$

Le cinéaste ne filme pas autre chose qu'un processus spiralaire où tout a été transformé sans que le centre n'ait bougé. C'est pourquoi le générique de fin reprend la musique du générique d'ouverture là où elle s'était arrêtée: on n'échappe pas au decrescendo. Ce regard pourrait renfermer bien du pessimisme, de la nostalgie. Mais il faut tendre l'oreille.

Pour lui [l'ingénieur du son pour le film] le son n'était pas une donnée brute qu'on subit et enregistre à partir de paramètres uniquement techniques, c'était une force à comprendre, à saisir, à capturer, à apprivoiser, à métamorphoser. En cela le son était bien la métaphore du monde entier, de la société tout entière, dont il n'acceptait pas non plus qu'elle soit donnée et inamovible. ${ }^{18}$
La bande sonore figure l'histoire en son mouvement. C'est la vertu de la forme du thème et variations qui va crescendo-decrescendo: on peut toujours décider de relancer le crescendo. Marker nous laisse la possibilité par la musique de composer nous-mêmes notre spirale, c'est-à-dire notre histoire. Il suffit que l'hymne soit ranimé pour retrouver le chemin des luttes:

J'ai essayé pour une fois (ayant en mon temps passablement abusé de l'exercice du pouvoir par le commentaire-dirigeant) de rendre au spectateur, par le montage, « son » commentaire, c'est-à-dire son pouvoir. ${ }^{19}$

Marker a conservé le pouvoir plus discret mais tout aussi efficace de la musique qui (lui) permet d'écrire l'histoire au présent. La musique n'est audible qu'à ceux qui l'écoutent comme le «son [de cette flûte] n'est perceptible qu'à celui qui en joue ${ }^{20} »$.

Regardons à nouveau le générique. Maintenant, on le perçoit, on l'entend: les lettres dessinent tout à la fois une portée et des notes, croches, noire (la masse circulaire), blanche (le « $\mathrm{O} »$ vide). Elles donnent la « clé » de la partition. Au seuil du film, le générique rappelle que l'histoire se lit et se voit autant qu'elle s'écoute.

17. Ibid.

18. Chris Marker, « Hommage à Antoine Bonfanti », Programme de la Cinémathèque de Corse, avril-juin 2005, p. 3. Texte daté du mercredi 29 octobre 2003.

19. Chris Marker, Le Fond de l'air..., p. 7.

20. C'est ce qu'écrit Marker, dès 1961, en exergue de ses Commentaires 1, ouvrage qui rassemble le texte et des images des documentaires qu'il a réalisés; il reprend cette formule, attribuée à Pouchkine, dans Sans Soleil (18'45”), qu'il réalise juste après Le Fond de l'air est rouge.

Vincent Casanova, «Écouter l'histoire ou la clé des luttes passées. Sur le générique du Fond de l'air est rouge, de Chris Marker (1977) » Écrire l'histoire, $\mathrm{n}^{\circ} 12$ - automne 2013, 75-81 